\title{
Proposed Equation of State Experimental Program at NOVA/NIF
}

N.C. Holmes, R. Cauble, P. Celliers, G. Collins, L. DaSilva, B. Hammel, R. Stewart, O. Strand and A. Sullivan

March 1996

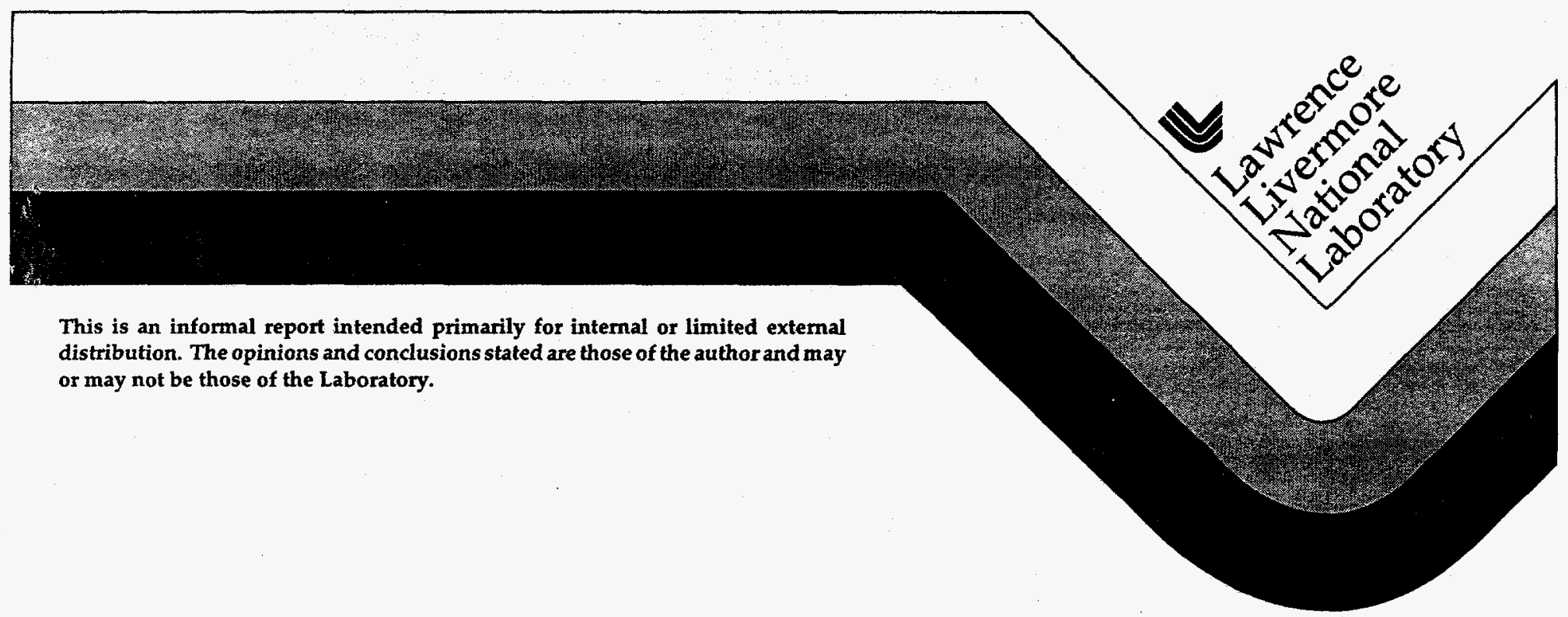




\section{DISCLAIMER}

This document was prepared as an account of work sponsored by an agency of the United States Government. Neither the United States Government nor the University of California nor any of their employees, makes any warranty, express or implied, or assumes any legal liability or responsibility for the accuracy, completeness, or usefulness of any information, apparatus, product, or process disclosed, or represents that its use would not infringe privately owned rights. Reference herein to any specific commercial product, process, or service by trade name, trademark, manufacturer, or otherwise, does not necessarily constitute or imply its endorsement, recommendation, or favoring by the United States Government or the University of California. The views and opinions of authors expressed herein do not necessarily state or reflect those of the United States Government or the University of California, and shall not be used for advertising or product endorsement purposes.

This report has been reproduced directly from the best available copy.

Available to DOE and DOE contractors from the Office of Scientific and Technical Information P.O. Box 62, Oak Ridge, TN 37831

Prices available from (615) 576-8401, FTS 626-8401

Available to the public from the

National Technical Information Service

U.S. Department of Commerce

5285 Port Royal Rd.,

Springfield, VA 22161 


\title{
Proposed Equation of State Experimental Program at NOVA/NIF
}

\author{
N. C. Holmes, R. Cauble, P. Celliers, G. Collins, L. Da Silva, B. Hammel, \\ R. Stewart, O. Strand, and A. Sullivan
}

\begin{abstract}
We sketch out a program to perform precise and accurate equation of state (EOS) experiments using large, high-power lasers. The program is divided into three phases: (1) a driver qualification program which will determine the necessary drive and target design parameters; (2) characterization of low and high-Z standard materials; (3) accurate impedance-match experiments to determine equations of state of materials of interest relative to the qualified standards. Novel methods are proposed for the first phase which are an order of magnitude more sensitive than those applied previously. We identify and describe the choices for standards and the samples slated for initial studies. In all cases, our goal is to obtain data that is of sufficiently high quality to serve for design purposes and to validate theories of material response at high pressures of the order of 1-10 TPa. This is particularly important right now, for materials such as $\mathrm{CH}$ plastics and hydrogen (DT). In addition, we will suggest a program for EOS measurements for $P>10 \mathrm{TPa}$, the region of maximum theoretical EOS uncertainty for many materials.
\end{abstract}

\section{Introduction}

The high pressure equations of state of materials must be accurately known for confident design, simulation, and interpretation of ICF target experiments and for weapons. This is particularly timely now, since our goals have shifted from testing to generating a predictive design capability. For example, the Laboratory will take part in substantial efforts toward new computational capabilities (ASCI); these efforts will ultimately fail to predict device performance without better, more extensive, and experimentally validated databases than now exist.

While Thomas-Fermi theory is valid for high- $\mathrm{Z}$ materials at ultra-high pressure and temperature, and impact experiments are available for $P \approx 0.1 \mathrm{TPa}$ ( $1 \mathrm{Mbar}$ ), the intermediate range of roughly $1-100 \mathrm{TPa}$ is difficult to treat theoretically and experimentally. With the end of nuclear-driven experiments, high intensity lasers may, and should, provide the best way to determine equations of state at extreme conditions. The Hugoniot equation of state is the most useful because it can be determined absolutely. The RankineHugoniot relations express the conservation of momentum, mass, and energy across the mov- ing shock front:

$$
\begin{aligned}
P-P_{0} & =\rho_{0} u_{s} u_{p} \\
V & =V_{0}\left(1-\frac{u_{p}}{u_{s}}\right) \\
E-E_{0} & =\left(P+P_{0}\right)\left(V_{0}-V\right) / 2
\end{aligned}
$$

where $E, P$, and $V$ are the total energy, pressure and volume. The initial density is $\rho_{0} \equiv$ $1 / V_{0}$, the velocity of shock propagation is $u_{s}$, and the material velocity behind the shock front is $u_{p}$. Subscripted (0) variables refer to initial conditions and unsubscripted to final states. The locus of $P$ and $V$ points satisfying equation 3 and originating from the same initial conditions is called the Hugoniot. If the initial density is known, then the determination of any two other variables serve to completely determine the final state. One other essential attribute of shock experiments is the boundary condition at interfaces between materials. When a shock passes from one material into another, $P$ and $u_{p}$ are conserved across the boundary. This property is exploited in the process of impedance-matching, shown schematically in Fig. 1.

The most straight-forward measurements are of the initial density $\rho_{0}$ and shock velocity $u_{s}$. Then we would need to find, for example, $\rho$ or $u_{p}$. This is difficult in most cases, and is 



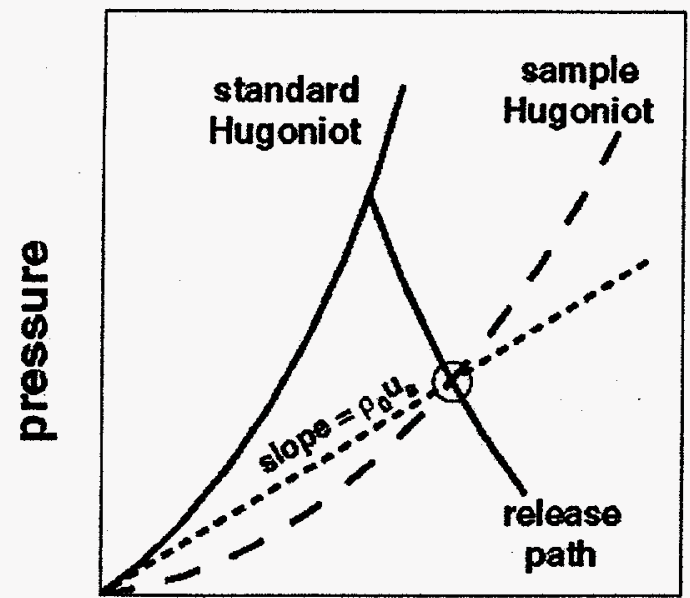

mass velocity

Figure 1: Schematic of the method of impedance matching. The values of $P$ and $u_{p}$ are continuous across the boundaries of adjacent materials. The values of shock velocity $u_{s}$ are measured in both the standard and the sample of unknown EOS. The EOS of the sample is determined relative to the equation of state of the standard.

certainly the problem with laser experiments as well. The usual approach is to determine, by some means, the EOS of "standard" materials, and then use the method of impedancematching to find the EOS of unknown materials relative to those standards. The process is depicted in Fig. 1 and described in section 3. This is the approach taken in most nuclear-driven EOS experiments, and these demonstrate the utility of multiple standards to "bootstrap" the EOS determination process. This is the approach we will use here. Our goal is to propose a program that exploits the convenience and reliability of the Hugoniot approach, and avoids the use of code simulations to determine the actual data.

Since the initial density is relatively easy to measure, we will not address that question here, although we note that it can be an issue for deposited metal foils. We will concentrate on the question of measuring shock velocity. Shock velocity will be determined by mea- suring the shock transit time $\Delta t$ of a steady shock wave across a step of known height $\Delta x$ and then $u_{s}=\Delta x / \Delta t$. This method, and the proposed use of impedance matching imply several conditions that all experiments must meet:

- The shock must be steady in time, i.e. $P$ and $u_{s}$ are constant across the step.

- The shock must be uniform and planar to assure equal drive across the known and unknown samples.

- The preheat must be negligible, or if not, must be small and known. This effects $E_{0}$ and $\rho_{0}$.

The program we propose provides, for the first time, a way to address all of these issues comprehensively. There are five parts to the program:

- determination of drive conditions for uniform and planar shock generation.

- a novel method for determining shock steadiness

- characterization of standard materials

- impedance-match experiments on $\mathrm{CH}$. $\mathrm{Au}$ is also interesting and needs to be done).

- Hugoniot experiments on liquid or solid $\mathrm{D}_{2}$ or DT.

\section{2 accuracy}

Before we go on the drive requirements, the accuracy we need to achieve has to be identified so that we can see how well the drive and target must be characterized. For example, the fractional uncertainty in final density $\delta \rho / \rho \sim\left(\rho / \rho_{0}-1\right) \delta u_{s} / u_{s}$. This places a premium on the accuracy of shock velocity measurements. While a complete treatment is beyond the scope of this proposal, some general guiding remarks can be made. 

Consider an average case of $\mathrm{Al}$ shocked to $50 \mathrm{Mbar}$ ( $5 \mathrm{TPa}$ ). The shock velocity is roughly $50 \mathrm{~km} / \mathrm{s}$, and the shock compression $\rho / \rho_{0} \approx 3$. Let's assume that we desire to measure $u_{s}$ to $1 \%$. For a step height of $100 \mu \mathrm{m}$, the transit time is 2 ns, which must be measured to at least $14 \mathrm{ps}$ absolute accuracy. This also means that the step height must be determined to better than $0.7 \mu \mathrm{m}$. Here we assume that the uncertainties in $\Delta x$ and $\Delta t$ are independent, and this is responsible for the factors of $1 / \sqrt{2}$. The fractional uncertainty in density will still be about $2 \%$. Certainly this is going to be very challenging, and is one of the main reasons for desiring to do such experiments at NIF, where the time and spatial scales can be larger than at Nova. However, these numbers seem well within the state-ofthe-art, so it makes sense to continue. Note that these experimental uncertainties made no assumptions about planarity or steadiness of the shock, but implicitly these have been assumed to add negligible error. We characterize these experiments as difficult but possible!

\section{Uniform drive}

We will emphasize the use of direct drive. While hohlraum drive is attractive for producing potentially spatially smooth drive, the problems of uniformity and $\mathrm{x}$-ray preheat, as well as a much more difficult diagnostic environment, encourage the use of simpler geometries. A typical experimental arrangement is shown in Fig. 2. Direct drive using phase plates to smooth the spatial extent of the beam, as well as the use of overlapping beams can produce the required $1 \%$ or so intensity uniformity over large spot sizes, on the order of several hundred $\mu \mathrm{m}$. We propose the use of $3 \omega$ drive to reduce preheat due to suprathermal electrons. This has been reliably demonstrated. Preheat issues will be addressed in section 4. Pulse shaping will be required to alleviate the 2-D plasma expansion effects at the ablation front.

In impedance-matching, we use the known EOS of the standard and the measurements of shock velocity in standard and sample. Since we know $u_{s}$ in the standard, we know the value of $P$ and $u_{p}$ at the standard-sample interface. This means that the values of $P, u_{p}$, and $u_{s}$ are then known in the sample and the EOS is determined. This is shown as the small circle in Fig. 1. Referring to Fig. 2, we require that the drive be uniform over a line corresponding to the equivalent image of the streak camera slit projected in the target plane, over the linear extent of the shock breakout area. This is needed to insure that pressure generated in the ablating layer, and the "standard" below the stepped samples is the same under both samples. This is smaller than the beam irradiation diameter due to edge rarefaction effects caused by the limited lateral extent of the laser beam. This is required for impedance-matching to work.

This can be characterized by observing the shock breakout from a plane-parallel sample disk with a streak camera at high time resolution. We propose to perform this characterization on two perpendicular axes across the back surface of the planar sample. Deviations from simultaneous shock breakout will be due to irradiation non-uniformity, assuming that the targets are homogeneous on a scale small compared to the resolution elements of the imaging system. We expect that some lateral smoothing will occur in the ablation layer and in the standard base material.

\section{Steady shock propagation}

That the shock must be steady is obvious; however, sufficiently sensitive tests have yet to be made to determine this. For accurate measurements, we wish the systematic error due to variations in shock velocity to be negligible compared to the measurement of shock velocity. While wedge tests on witness plates 


\section{$\bigwedge \begin{aligned} & \text { streak } \\ & \text { camera }\end{aligned}$}

\section{standard unknown}

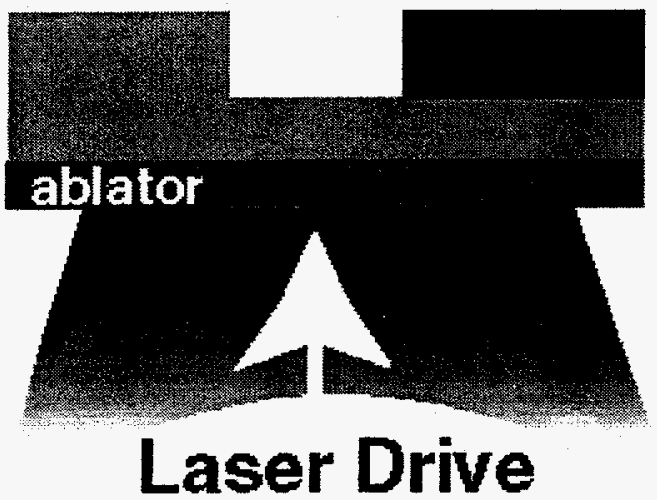

Figure 2: In the figure, the shock arrives from the bottom and is equal in strength at arrival at both steps. Shock velocities are measured in the sample and "standard" materials. The value of $u_{s}$ in the standard is used to determine the strength of the shock arriving at the sample. continue to be performed, they suffer by comparison the novel method we propose.

Consider the passage of a slightly nonsteady shock across a wedge or step. From the Hugoniot equations above, we see that for strong shocks $P \approx \rho_{0} u_{s}^{2}$. This means that measurements of time (as in wedge tests), are sensitive to $\sqrt{P}$. A more sensitive test, which is performed, incidentally, in temperature measurements at LLNL's two-stage gun, measures the intensity of the emission from the shock front itself, moving in an initially transparent medium. We see that

$$
I \approx \sigma T^{4} \sim E^{4} \sim P^{4} \sim u_{s}^{8}
$$

Thus, small changes in shock velocity or pressure are much more sensitively recorded by observing the shock emission. We have developed a fiber optic method which is both highly sensitive (equivalent $\mathrm{f} \#$ is about 2.5), and free of geometric or depth of field effects. This statement does not hold for the SOP now in use. Furthermore, it is not necessary to absolutely calibrate the system to determine temperature-only the time dependence is of interest here. Direct drive environments will be more conducive to doing this experiment as well, since shielding requirements are less. The experiment will just consist of a fiberoptic probe placed in close proximity to the sample, and an accurately calibrated streak camera to record the time dependence of the signal. The sample must be initially transparent, so we will want to use a three layer target: ablator, a thin opaque layer such as $\mathrm{Au}$, and an $\mathrm{SiO}_{2}$ sample. This will serve as a test bed to validate simulations of steady drive, and this capability will be needed for other experiments.

\section{Preheat}

Since the system described above will be highly sensitive, it may also serve to determine the actual preheat levels in the target if 

the recording system is calibrated to a standard source. Preheat is a potentially serious issue. Preheat will raise the initial energy $E_{0}$, so that the sample is no longer on the principal Hugoniot. For very strong shocks, for which $E \gg E_{0}$, this is not very serious. However, strong preheat levels can also decrease $\rho_{0}$ due to thermal expansion. The latter is the most serious problem, and must be eliminated. To give some feeling for how severe this issue is, consider some typical metals that may be used in our experiments. Aluminum has a thermal expansion coefficient at STP of $25 \times 10^{-6} /{ }^{\circ} \mathrm{K}$. At the melting temperature of about $900 \mathrm{~K}$, we can estimate a linear expansion of roughly $2.3 \%$. Since $d \rho / \rho=-3 d x / x$, the initial density has changed by about $7 \%$ with a preheat level less than $0.1 \mathrm{eV}$ ! This is typical of metals, which are typically about $10 \%$ expanded at melt. Clearly, we must find a way to determine the preheat levels at temperatures which are below the point for which the material has significant optical luminosity. Recent experiments at AWE are the first to be precise enough to indicate a systematic deviation from expected values in $\mathrm{Al} / \mathrm{Cu}$ impedance-matching experiments. The observed discrepancy may well be due to uncharacterized low levels of $\mathrm{x}$-ray preheat.

It should be remembered that the thermal expansion is not instantaneous. It proceeds as a rarefaction wave moving in from the free surfaces at the sound velocity (for $\mathrm{Al}$ $c_{B} \approx 5 \mathrm{~km} / \mathrm{s}=5 \mu \mathrm{m} / \mathrm{ns}$ ). In the rarefied region, the density is less, and one can expect $u_{s}$ and $u_{p}$ to differ from their values on the principal Hugoniot. This means that the shock may not be steady because the shock traverses regions of varying density. In addition, the insertion of preheat shields in the interior of the targets may also affect the hydrodynamics: a preheated high- $Z$ layer may drive shocks into the surrounding materials, perturbing the initial state.

A recent suggestion by $\mathrm{G}$. Collins is to use
anti-Stokes Raman methods to determine the preheat levels in molecular materials such as $\mathrm{D}_{2}$ or $\mathrm{SiO}_{2}$. This may well be a fruitful approach. In addition, time-resolved interferometric methods may be used to determine the motion of the free surface during preheat. In all of this, it's important to mention that the physics of preheat by $x$-rays or electrons involve the kinetics of energy transfer from electrons to phonons, and this fact must also influence our notions of preheat characterization.

\section{6 standards}

We propose to use the technique of impedance-matching, so that the equationsof-state of test samples will be measured by comparison with materials whose EOS is believed to be well-known. The standard materials must satisfy several criteria: they must be easily available in pure form, they must be easily made or machined to sub-micron tolerances without change in bulk properties, they must be stable in phase up to melt, or well-known otherwise, they must be able to be confidently and accurately modeled using our best available EOS theories, and, finally, their EOS must be known over a wide range of pressure and temperature.

The materials that best suit these criteria are transition metals: $\mathrm{Al}, \mathrm{Cu}, \mathrm{Mo}, \mathrm{Pb}$, $\mathrm{Ta}, \mathrm{Au}, \mathrm{Pt}$. Some of these have been characterized in nuclear-driven EOS experiments. We believe the best current choice is aluminum. It is a nearly-free-electron system, and is well suited to modern theoretical treatments. It has been used as the LLNL standard for nuclear-driven experiments, and we believe that its properties are well known up to about $25 \mathrm{Mbar}$.

We propose a series of experiments on $\mathrm{Al}$ to serve as comparisons with existing data and theory. Agreement of the laser-driven data will serve to establish it for our use at extreme 

pressures. We will use the Al standard to fur-. ther qualify other standard materials, such as $\mathrm{Au}$ or Pt.

\section{Impedance-match experiments}

We plan a series of experiments to determine the Hugoniot EOS of $\mathrm{CH}$ in pure and bromineloaded forms. These are of particular interest to the ICF diagnostic and instability program. The EOS of $\mathrm{CH}$ at pressures below $1 \mathrm{Mbar}$ and $1 \mathrm{eV}$ is dominated by the phase equilibria of carbon, and the best available theory (CHEQ) may not be useful in the short time scale and high temperatures of laser experiments. Previous EOS tables of $\mathrm{CH}$ properties are derived from the TIGER EOS code, and are benchmarked by high explosive data, again outside the range of usefulness for our needs. Thus we will determine the EOS of $\mathrm{CH}$ by comparison with the $\mathrm{Al}$ standard. We welcome suggestions from the ICF program to help define the materials and short-term goals of this phase of the program.

\section{Compression of deuterium}

One of the most important materials in the ICF program is deuterium. However, the current situation is that there is large uncertainty in the EOS in the roughly 1-10 Mbar range, depicted in Fig. 3. This is well above the gasgun single-shock limit of $\mathbf{3 0 0 ~ k b a r , ~ a n d ~ a l s o ~ i s ~}$ in a regime for which the most used models, QEOS for example, are simply wrong or do not use appropriate physics. Obviously, better theory is needed, and the situation in the pressure range of interest is complicated by molecular dissociation and ionization. First principles calculations are possible but are extremely time-consuming and difficult to extrapolate. Experiments are needed.

We envision a layered target consisting of the shock generator layer(s), an Al baseplate, a liquid or solid $\mathrm{D}_{2}$ sample, and a transparent

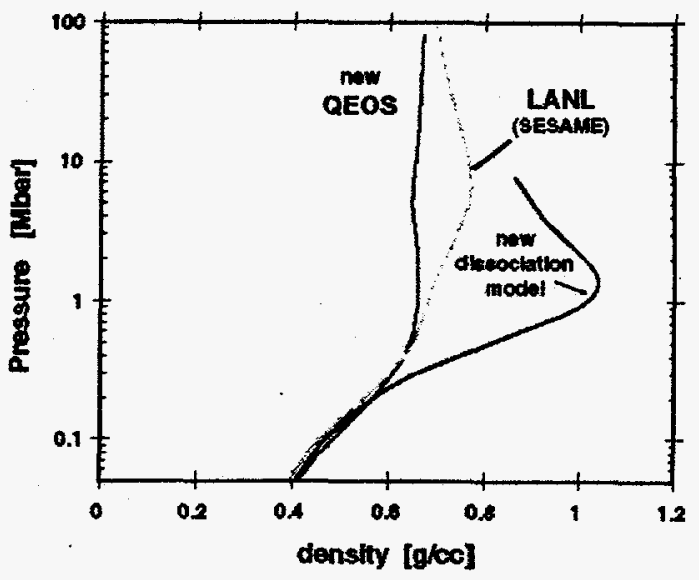

Figure 3: Plots of the Hugoniot of liquid $D_{2}$. QEOS was modified in an attempt to include molecular dissociation and was used to calculate the left-most curve. The mixture model of $M$. Ross was used to calculate the right-most curve. The SESAME EOS, which agrees with the values in the LLNL EOS tables obtained with QEOS, falls between the two extremum curves. Neither the SESAME tables nor the earlier QEOS-generated table agrees with recent shock temperature data.

window, as depicted in Fig. 4. The shock velocity in the $\mathrm{Al}$ standard is measured across the step, as usual, and the shock velocity in the $D_{2}$ is measured across the step in the window. Here we make use of the fact that the $D_{2}$ is reshocked at the window interface, and the increase in intensity is recorded by the streak camera. For experimental accuracy, we prefer to use liquid $D_{2}$, since the density is simply related to the saturation pressure, and the sample is homogeneous. Then the step is easily defined by the window and the $\mathrm{D}_{2}$ needs no machining. Solid $\mathrm{D}_{2}$ may also be used, but care must be taken to avoid bubbles, and the density must be well-known and at full solid density.

However, we note that theory is needed to calculate the $\mathrm{Al}$ release isentrope, and we must have confidence in the thermal EOS in the theory to do this. This may not be a problem, and we can certainly test it with impedance-match experiments on porous $\mathrm{Al}$ 


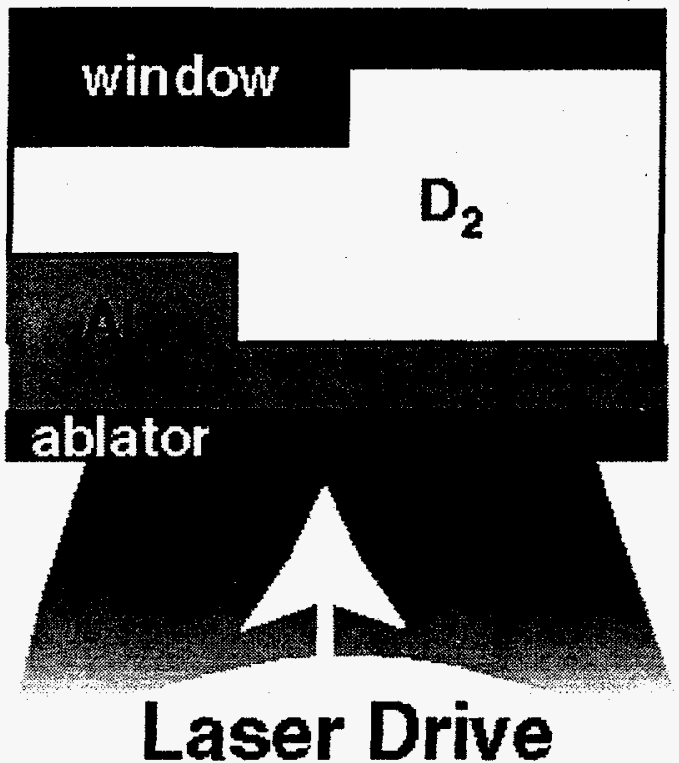

Figure 4: A suggested experimental layout for a liquid $\mathrm{D}_{2}$ experiment.

in the pressure and temperature range of interest.

\section{EOS at $P>100 \mathrm{Mbar}$}

At very high pressures, above about $100 \mathrm{Mbar}$ in metals, the EOS needs are somewhat different. While still in the intermediate range between the $\approx 1$ Mbar data and Thomas-Fermi, here the EOS is dominated by thermal effects and ionization. Parenthetically, this is also the case for hydrogen at $1 \mathrm{Mbar}$. Since the Hugoniot equations do not explicitly give the temperature, the partition of shock energy between internal modes is unknown. However, theories of eos and opacity in this regime do often explicitly use temperature as an independent variable. When the available models are extrapolated into this region of EOS space, we often find that, while fairly good agreement with $P$ and $\rho$ is found, temperature can be uncertain by a factor of two. This is a serious problem, and will impact our ability to model opacity. We recommend a study of temperature experimental methods. $\mathrm{X}$-ray spectroscopy should be investigated as a means to determine temperature.

\section{Errors}

The subject of accuracy of EOS experiments with regard to distinguishing between theory was briefly discussed recently. I thought it might be useful for some of you to run through an analysis on the propagation of errors in EOS measurements. We start with the Hugoniot relations, above Eqs. 1-3, and neglect the initial pressure and energy on the lefthand side of the equations. By taking total derivatives and a little algebra, it is easy to show that

$$
\frac{d P}{P}=\frac{d \rho_{0}}{\rho_{0}}+\frac{d u_{s}}{u_{s}}+\frac{d u_{p}}{u_{p}}
$$

and

$$
\frac{d \rho}{\rho}=\frac{d \rho_{0}}{\rho_{0}}-(\eta-1) \frac{d u_{s}}{u_{s}}+(\eta-1) \frac{d u_{p}}{u_{p}}
$$

where $\eta=u_{s} /\left(u_{s}-u_{p}\right)=\rho / \rho_{0}$ is the compression behind the shock front.

If we assume that we make independent measurements of $\rho_{0}, u_{s}, u_{p}$ with uncertainties $\delta \rho_{0}, \delta u_{s}, \delta u_{p}$, respectively, then the uncertainties in the results will be just the r.m.s. sum of the errors of the individual measurements:

$$
\begin{aligned}
\left(\frac{\delta P}{P}\right)= & {\left[\left(\frac{\delta \rho_{0}}{\rho_{0}}\right)^{2}+\left(\frac{\delta u_{s}}{u_{s}}\right)^{2}+\left(\frac{\delta u_{p}}{u_{p}}\right)^{2}\right]_{(7)}^{1 / 2} } \\
\left(\frac{\delta \rho}{\rho}\right)= & \left\{\left(\frac{\delta \rho_{0}}{\rho_{0}}\right)^{2}\right. \\
& \left.+(\eta-1)^{2}\left[\left(\frac{\delta u_{s}}{u_{s}}\right)^{2}+\left(\frac{\delta u_{p}}{u_{p}}\right)^{2}\right]\right\}^{1 / 2}(8)
\end{aligned}
$$

The factor of $\eta-1$ is easily understood. In Eq. 2, $\left(1-u_{p} / u_{s}\right)^{-1}$ can be rewritten as $u_{s} /\left(u_{s}-u_{p}\right)$. For large compressions, $u_{s}$ and 
$u_{p}$ must have similar values and the difference between the two variables will be most sensitive to error when their difference is low. The upshot is that we must make very careful measurements, no matter what!

Let's consider a real case for $\mathrm{D}_{2}$ at $3 \mathrm{Mbar}$. The mass velocity is $x \mathrm{~km} / \mathrm{s}$, the shock velocity is $y$, and the initial density is $0.171 \mathrm{~g} / \mathrm{cm}^{3}$. Assume measurement errors of $2 \%$ in $u_{s}$ and $u_{p}$, and $1 \%$ in density. Then the total expected uncertainties on pressure and density are:

$$
\begin{aligned}
& \left(\frac{\delta P}{P}\right)=3 \% \\
& \left(\frac{\delta \rho}{\rho}\right)=5.9 \%
\end{aligned}
$$

- For the case where $u_{p}$ is not measured directly, we must use impedance matching to find its value by comparison with a standard such as aluminum or copper. In this case, the uncertainty in $u_{p}$ arises from measurement errors of $u_{s}$ in the standard, and from systematic uncertainty in the Hugoniot of the standard. In practice, we estimate this systematic uncertainty over some range of $u_{p}$ by fitting the actual data and Hugoniot analytic fit to a parabolic model, and introduce three coefficients $A_{0}, A_{1}, A_{2}$ to represent the total systematic uncertainty. A parabolic model is appropriate to linear Hugoniot fits, since the uncertainty in the fit is higher at either end. To be explicit, we say that the experimental and systematic uncertainties in $u_{p}$ for the standard with a linear Hugoniot $u_{s}=C+S u_{p}$ are given by:

$$
\begin{aligned}
& \left(\frac{\delta u_{p}}{u_{p}}\right)_{\exp }=\frac{1}{S}\left(\frac{\delta u_{s}}{u_{s}}\right) \\
& \left(\frac{\delta u_{p}}{u_{p}}\right)_{\text {sys }}=A_{0}+A_{1} u_{p}+A_{2} u_{p}^{2}
\end{aligned}
$$

and the total uncertainty in $u_{p}$ is given by

$$
\left(\frac{\delta u_{p}}{u_{p}}\right)=\left[\left(\frac{\delta u_{p}}{u_{p}}\right)_{\exp }^{2}+\left(\frac{\delta u_{p}}{u_{p}}\right)_{\mathrm{sys}}^{2}\right]^{1 / 2}
$$

This implies that a very good $u_{s}$ measurement is much better than a mediocre $u_{p}$ measurement, as long as the standard is well known. Since we have no absolute data for any material above a few Mbar, this can be a real problem. It is not insurmountable, since we do have good impedance match data and excellent theory for simple metals up to tens of Mbar from nuclear experiments in the U.S. and Russia.

\section{1 acknowledgments}

This work was performed under the auspices of the U.S. Department of Energy by the Lawrence Livermore National Laboratory under Contract No. W-7405-ENG-48. 



$$
-1
$$

